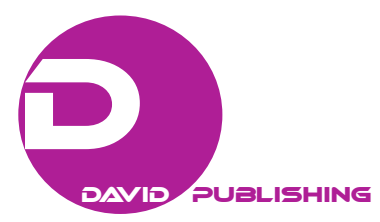

\title{
Storage Efficiency As a Managerial Optimization Problem*
}

\author{
Simona Hašková, Robert Zeman \\ Institute of Technology and Business, České Budějovice, Czech Republic
}

\begin{abstract}
The paper derives efficient methods for solving basic fundamental tasks of warehouse logistics in what amount and when to resupply the stocks by means of the "case-based reasoning" approach. The aim is to give a satisfactory response, including its economic interpretation under conditions of uncertainty. Therefore, the discrete case is first studied in detail by means of the tools of microeconomic analysis and accomplished results are then generalized by the limiting transition to the continuous case. In both cases, the discreet and continues one, the method relies on the value of the newly established indicator of the storage efficiency, which indicates that what inventory items are worth storing in the case of the limited storage capacity. Its value depends on the fragment of the probability distribution of the demand during the delivery time.
\end{abstract}

Keywords: logistics, daily demand, delivery time, loss of margin, storage efficiency indicator, probability distribution

\section{Introduction}

In practice, most sellers or manufacturers, who cannot manage without storing the retailed goods or components required in manufacturing, are looking for the right answer to the question "in what quantity and when to resupply their stock". The criteria of "the correct" answer here is the minimization of the total cost associated with it (Williams \& Tokar, 2008). The parameters, on which the correct answer depends, are:

- $D D$ - the variable daily demand with the $D D$ values showing the number of removed units of the considered inventory items from storage in one working day of the current year;

- $D$ - the variable annual demanded consumption of the considered inventory items with the values $D=$ $\Sigma D D_{i}$ (summing up all the working days of the current year);

- $H$-the variable part of the annual costs associated with the storage of one unit of the inventory item;

- $K$-costs associated with one supply of the inventory item (ordering, transferring, and handling costs of one delivery that within given specific logistic terms do not depend on the number of $X$ units of supplement delivery);

- $D T$ - the variable delivery time of the ordered additional supply (its values $D T$ indicate the number of

\footnotetext{
* Acknowledgements: The publishing costs of this contribution are covered by the Department of Economics and Management, Faculty of Management, Institute of Technology and Business, Okružní 517/10, 37001 České Budějovice, Czech Republic.

Simona Hašková, Ing., Department of Economics and Management, Faculty of Management, Institute of Technology and Business, České Budějovice, Czech Republic.

Robert Zeman, Ing., Ph.D., Department of Economics and Management, Faculty of Management, Institute of Technology and Business, České Budějovice, Czech Republic.

Correspondence concerning this article should be addressed to Simona Hašková, Department of Economics and Management, Faculty of Management, Institute of Technology and Business, Okružní 517/10, 37001 České Budějovice, Czech Republic. E-mail: haskovas@post.cz.
} 
working days that pass from placing the order of the additional supply to its acceptance into stock);

- $L$ - the loss from each considered inventory item that would not satisfy the current demand due to an empty storehouse (the loss from customer dissatisfaction in the case of a retailer or the failure of production in the case of a manufacturer, e.g. the lost margin).

If authors assume from the above unlimited time duration of business, it is customary to consider the possible variants within one accounting period, which is usually a calendar year. It is also suitable to split the initial question into two questions: "how much to reorder" and "when to reorder". If $X$ is the answer to the question "how much to reorder", then the values of the variable $D / X$ are the annual numbers of orders of additional supply. If it denotes by $R L$ (reorder level) the level of the considered inventory item $(R L \geq 0)$, at which level the storehouse gives the order for an additional supply, then authors can answer the question "when to reorder" by pointing to the selected $R L$.

In the following, this paper proceeds from the standard textbook model (see Brealey, Myers, \& Marcus, 2011) and precises the solution in terms of certainty. It gives reasons for the adaptation and generalization of this model on conditions of uncertainty and then by the "case-based reasoning" approach, it derives a general procedure for solving the task.

\section{Model and the Solution of the Problem in the Case of Even Storage Removal in Terms of Certainty}

Ideally, in the case of even storage removal where $D D$ and $D T$ are the constants ( $D D=d d$ and $D T=d t)$, the dynamics of stocks (i.e. the course of the state of stock, depending on the time measured by the number of working days) can be approximated by the continuous model, which is the "saw" in Figure 1. This is exactly valid, for example, for the state of stock of even flowing fluid refilled to the initial level of $X$ at the moment of depletion of the tank (Chen, Ray, \& Song, 2006).

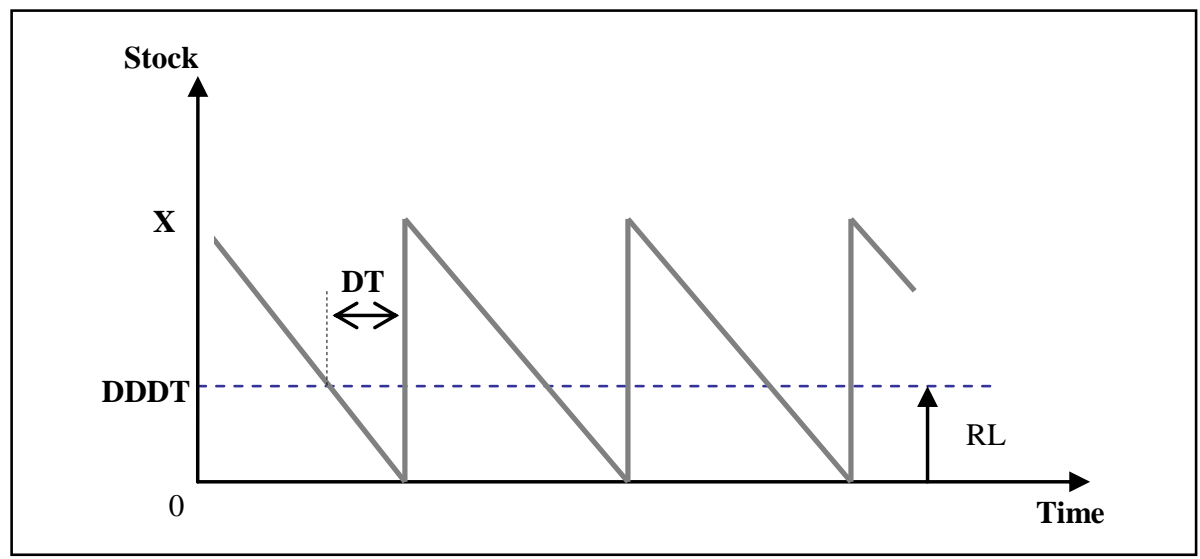

Figure 1. Dynamics of stock in the ideal case.

The variable $D D D T$ (the demand during the delivery time) is given by the product of $D D D T=D D \times D T$, and therefore in this case, it has a constant value $D D D T=d d d t=d d \times d t$. For $R L_{o p t}$ (i.e. $R L$ minimizing the total costs associated with the storage of the inventory item and thus correctly answering the question "when to reorder"), it applies that $R L_{\text {opt }}=D D D T$ independently of $X \geq D D D T$.

It is obvious that the deviation of the $R L$ value from the value $D D D T$ on this or that side results in the increase of loss (costs) associated with the storage of the inventory item. Therefore $R L_{o p t}=D D D T$. If $R L=$ 
$D D D T$, then the resulting dependence of the value of the total annual costs $N(X)$ associated with the storage of the inventory item on the amount $X$ of the additional supply can be described by:

$$
N(X)=H \times X / 2+K \times D / X
$$

where $H \times X / 2$ is the annual costs of storing and $K \times D / X$ is the annual amount of ordering, transferring, and handling costs associated with the replenishment of stock. If it denotes $E O Q$ (economic order of quantity) as the volume of the supplemental supply (i.e. the value of variable $X$ ), minimizing the expression (1) (Maddah \& Jaber, 2008), then EOQ can be obtained by solving the system $d N(X) / d X=H / 2-K \times D / X^{2}=0, d^{2} N(X) / d X^{2}=2$ $\times K \times D / X^{3}>0$ due to the variable $X$ :

$$
E O Q=(2 \times K \times D / H)^{1 / 2}
$$

By substituting $E O Q$ for $X$ in (1) and by the subsequent substitution of the expression $(2 \times K \times D / H)^{1 / 2}$ for $E O Q$ to the right side of the equation, the following can be successively got:

$$
N(E O Q)=H \times E O Q / 2+K \times D / E O Q=(2 \times K \times D / H)^{1 / 2}
$$

\section{Adaptation of the Model to the Conditions of Uncertainty}

In the examined ideal case (Figure 1) when $R L=D D D T$, the last unit of the inventory item is contracted out at the time of arrival of a new supply (Shapiro \& Wagner, 2009; Wokurka \& O'Leary-Kelly, 2000). If $R L>$ $D D D T$, then the decrease of its stock would regularly stop at the level $S L=R L-D D D T$. For the value $S L$, the teeth of the "saw" shown in Figure 1 (starting from the second tooth) would move upward. The level of $S L$ is a safety stock, which in a case of need (an unexpected increase in the value of $D T$ or an unexpected occurrence of $d d_{i}>d d$ within the time of delivery) acts as a buffer contained in $R L$. Its aim is to eliminate partially or completely the threat of customer dissatisfaction, due to the unexpected presence of DDDT above the usual level. In the described ideal case, where this is excluded, this buffer is not needed. It would only increase the storage cost by $H \times S L$. Therefore, in Figure $1, S L=0$ is selected.

The practice sometimes substantially deviates from the examined ideal case. Both the daily demand $(D D)$ and the length of delivery time $(D T)$ may vary over time and their fluctuations make their product variable, thus the value of the variable $D D D T$. If values of $D D$ and $D T$ can, in this context, be regarded as independent random variables with known probability distributions of their values, then $D D D T$ will be the random variable with probability distribution inferred from the distribution of variables $D D$ and $D T$. Under certain assumptions, this paper can consider every "saw teeth" from the perspective of a long time horizon as the results of repeating of the same random process consisting of elementary random experiments that take place within individual working days. If a number of repetitions is large enough, so that the law of large numbers could be applied (Jiroušek \& Kushmerick, 1997), this paper can represent the variable $D D, D T$, and $D D D T$ as the unbiased predictions of their values, i.e. as the expected values $E(D D), E(D T)$, and $E(D D D T)$. Completely analogous to Figure 1 is then Figure 2.

In it (unlike Figure 1), the safety stock level $S L$ is an essential part of $R L$ used to eliminate the unavoidable overhangs of the variable values $D D D T$ over $E(D D D T)$. Therefore, it makes sense to consider $S L$ only within the limits $0 \leq S L \leq\left(d d d t_{\max }-E(D D D T)\right)$, where for $R L$ applies $E(D D D T) \leq R L \leq d d d t_{\max }$. This model can, in a certain sense, be regarded as a statistical average of all possible random realizations of a long-term process of "development inventory stock" with the additional supply in the amount of $X_{\text {opt }}$ units and with $R L=E(D D D T)$ $+S L$. For determination $X_{\text {opt }}$ (the correct answer to the question "how much to reorder") of this model, it applies all that have already been mentioned in connection with the model in Figure 1. Much more difficult is finding 
$R L_{\text {opt }}$, i.e., the correct answer to the question "when to reorder" (Wei \& Chen, 2009). The following part of this paper deals with the solution to this problem.

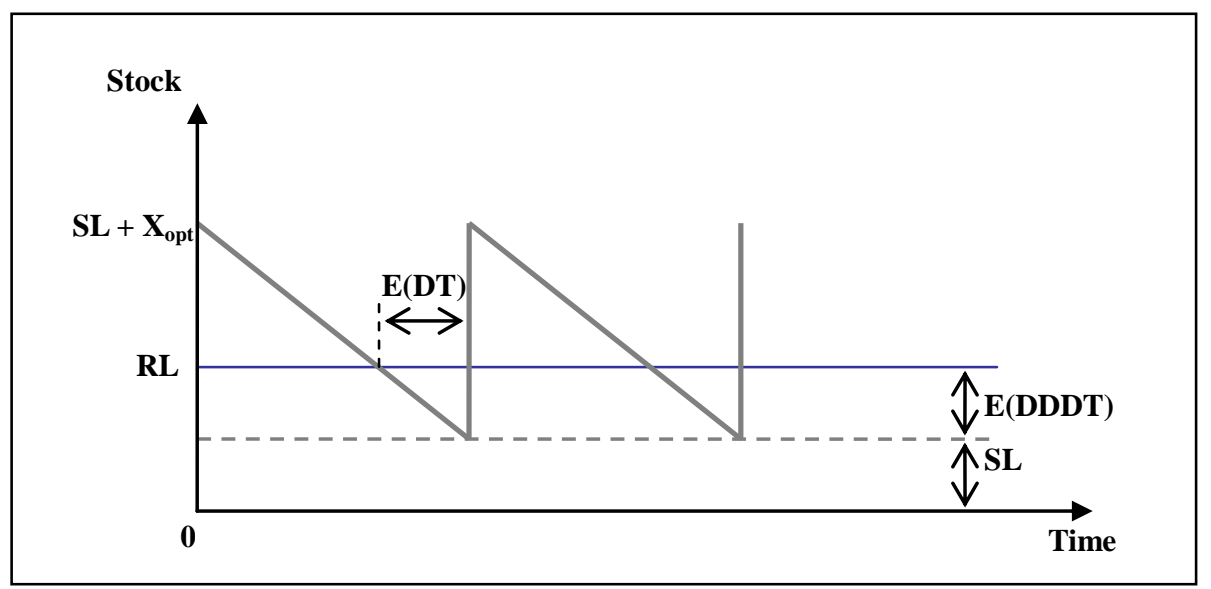

Figure 2. Model of dynamics of stock in terms of uncertainty.

\section{General Solution of the Problem by the "Case based Reasoning" Approach}

Both considered models here (i.e. the model in terms of certainty in Figure 1 and the model in terms of uncertainty in Figure 2) answer the question "how much to reorder" in the same way. In both cases, authors obtain $X_{\text {opt }}$ by the procedure described in Article 2. The certain annual consumption of $D$ will be replaced by the expected value $E(D)=E\left(\Sigma d d_{i}\right)$ in terms of uncertainty. The same thing can be said of the total annual cost (1), induced by the "saw" component of the examined fluctuations of the stock. The only difference is that while in the first case it deals with the real costs, in the second case it deals with the expected cost (Chen et al., 2006).

The source of the substantial difference between the two cases is the existence of the expected annual cost $E(N S L(R L))$ of the safety stock that depends on the chosen $R L$ level under conditions of uncertainty (Federgruen \& Heching, 1999). By analogy with equation (1), valid in terms of certainty, at $X=X_{\text {opt }}$ for the expected total cost $E(N(R L))$, it can be written:

$$
E(N(R L))=H \times X_{o p t} / 2+K \times E(D) / X_{o p t}+E(N S L(R L))
$$

In the general case, $E(N S L(R L))$ has two components: the expected annual storage costs of the safety stock level in the amount $H \times S L=H \times(R L-E(D D D T))$ and the expected annual loss $E(L L S(H D))$ from a lack of stock. Therefore,

$$
E(N S L(R L))=H \times S L+E(L L S(R L))
$$

To minimize $E(N(R L))$, according to $R L$, means to minimize $E(N S L(R L))$, according to $R L$. In the following, it derives an algorithm to minimize $E(N S L(R L))$, according to $R L$ in the discrete case and then it generalizes the idea of a solution to the continuous case.

\section{Minimizing $E(N S L(R L))$ in the Discrete Case}

By "the discrete case", it means the case when the unit of inventory is no more arbitrarily divisible (e.g. the washing machine in the warehouse store of washing machines, etc.). If assuming $R L_{\max }=d d d t_{\max }$, then $E\left(N S L\left(R L_{\max }\right)\right)=H \times S L_{\max }=H \times\left(d d d t_{\max }-E(D D D T)\right)$. Because there is no shortage of stock, $E\left(L L S\left(d d d t_{\max }\right)\right)$ $=0$. A gradual reduction of $R L$ from $R L_{\text {max }}$ to $R L-1$ gradually decreases $S L$ to $S L-1$ (see Figure 2). This leads 
to a constant marginal saving of the annual storage costs of safety stock by $H \times S L-H \times(S L-1)=H$. At the same time, it comes to a gradual increase of the marginal expected annual losses from the lack of stock by

$$
\Delta E(L L S(R L))=E(L L S(R L-1))-E(L L S(R L))
$$

The idea of the algorithm of minimization $E(N S L(R L))$ can be described as follows: As the first current value $R L$, it chooses the value equal to $d d d t_{\max }$ and then for each current $R L$, it counts according to (6) $\triangle E(L L S(R L))$. If $\Delta E(L L S(R L)) \geq H$, then by the transition to $R L-1$, it is no better off. Thus $R L_{o p t}=R L$ and the process ends. In the opposite case when $\Delta E(L L S(R L))<H$, the new current $R L$ becomes $R L-1$ and the calculation according to (6) is repeated for the new current $R L$. The flowchart of the algorithm of this idea, in which the designation $\triangle E(R L)$ is an abbreviation for $\triangle E(L L S(R L))$, is shown in Figure 3.

The algorithm elaborates from $R L_{\text {max }}$ to $R L_{\text {opt }}$ from above and $R L_{\text {opt }}$ is the one $R L$, to which the algorithm arrived by its recursive steps, and that as a result of (6) satisfies the condition

$$
\Delta E(L L S(R L)) \geq H \geq \Delta E(L L S(R L+1))
$$

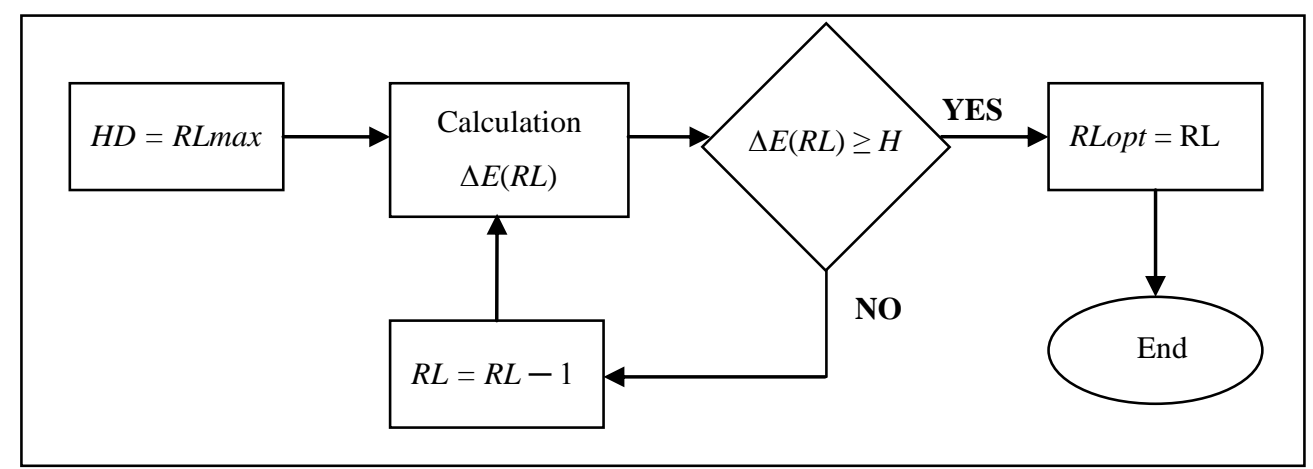

Figure 3. The flowchart of the optimization algorithm idea.

The calculation of $E(L L S(R L))$ for various $R L$ is based on the probability distribution of the discrete random variable $D D D T$ and on other terms, which are therefore necessary to define:

- Given a set $\Omega=\left\{0,1, \ldots, d d d t_{\max }\right\}$ of integer values of the variable $D D D T$ and the projection $p: \Omega \rightarrow\langle 0$, $1\rangle$ that for the sum of $p(i)$ over all $i \in \Omega$ holds $\Sigma p(i)=1$. The projection $p$ defining the set of pairs $\{(i, p(i)): i \in$ $\Omega$, where $i$ is the current value of $d d d t$ and $p(i)$ is probability occurrence, is called probability distribution of a discrete random variable $D D D T$;

- The excess of demand over the $R L$ is a discrete random variable $E D(R L)$ with $e d$ values dependent on the values of $d d d t$, for which it follows: $e d=0$ for $d d d t=i \leq R L$ and $e d=i-R L$ for $d d d t=i>R L$. Hence for the value $e d=0$, it gets (summing in the range from $i=0$ to $i=R L$ ) its probability occurrence of $\Sigma p(i)$; for $e d=i-$ $R L$, it gets the probability occurrence of $p(i)$. Furthermore, it is obvious that the expected value of the demand excess $E(E D(R L))=0 \times \Sigma p(i)+\Sigma(i-R L) \times p(i)=\Sigma(i-R L) \times p(i)$, where in the result it is summed over all $i>$ $R L$;

- $E(L L S(R L))=E(E D(R L)) \times L \times E(D) / X_{o p t}$, from which after substitution to (6) and after adjustment, it gets $\Delta E(L L S(R L))=(E(E D(R L-1))-E(E D(R L))) \times L \times E(D) / X_{\text {opt }}=(\Sigma p(i)) \times L \times E(D) / X_{\text {opt }}$, where in the result, it is summed over all $i \geq R L$. Analogously, $\Delta E(L L S(R L+1))=(\Sigma p(i)) \times L \times E(D) / X_{\text {opt }}$, where in the result, it is summed over all $i \geq R L+1$. Substituting both results in (7), it obtains after adjustment:

$$
p(R L)+\Sigma p(i) \geqslant H \times X_{o p t} / L \times E(D)>\Sigma p(i)
$$


where in both summations, it is summed over all $i>R L$. Sought $R L_{o p t}$ is the $R L$, which satisfies the condition (8). This condition can be made more transparent by designation of the constant $H \times X_{o p t} /(L \times E(D))$ by the symbol $\Phi$, by which it takes the shape of

$$
p(R L)+\Sigma p(i) \geqslant \Phi>\Sigma p(i)
$$

The constant $\Phi=H \times X_{\text {opl }} /(L \times E(D))$, sandwiched in (9) among the inequalities is the inverse value of the efficiency storage indicator.

$$
\Psi=L \times E(D) / H \times X_{\text {opt }}=(\mathrm{L} / H) \times\left(E(D) / X_{\text {opt }}\right)
$$

This indicator is the product of the ratio of the unit loss from the lost margin to the annual cost of the unit storage $(L / H)$ and the annual number of orders $\left(E(D) / X_{o p t}\right)$. It measures the loss of the dissatisfaction of the annual consumption of the inventory item with the annual costs of the storing of the optimum additional supply. The condition (9) in combination with Figure 2 then says that the higher this indicator is (and therefore $\Phi$ lower), the higher the safety stock level is worth holding. In the case of the limited storage capacity, it can be decided on the basis of the analysis of indicators, which are worth storing and which are not.

\section{Minimization of $E(N S L(R L))$ in the Continuous Case}

By the continuous case, it means that the unit of stock is arbitrarily divisible (as it is in the case of coal in the boiler room, the fluid reserve in the reservoir, and etc.). The transition from discrete to continuous case can be reached by the "from below" unbounded physical division of the original unit. In terms of mathematical analysis, it corresponds to the possibility of the limit transition from differential $\Delta x=1$ in the discrete model to an arbitrarily small value of the differential $d x \rightarrow 0$ in the continuous model. This is related to the transition from a finite number of elements in the set of values $\Omega=\left\{0,1, \ldots, d d d t_{\max }\right\}$ of the discrete random variable $D D D T$ to the interval $\left(0, d d d t_{\max }\right)$ of countless values of $d d d t$ of the continuous variable $D D D T$.

The initial distribution $p: \Omega \rightarrow(0,1)$ of the discrete $D D D T$ in the form $\{(i, p(i)): i \in \Omega\}$, where $p(i)$ is the probability occurrence of $d d d t=i$, passes in the case of the continuous DDDT in the continuous function $f:(0$, $\left.d d d t_{\max }\right) \rightarrow(0,1)$, in which for each $x \in\left(0, d d d t_{\max }\right)$ and for a sufficiently short interval $d x \subset\left(0, p b d d_{\max }\right)$, the product of $f(x) d x$ gives the probability that $x \leq d d d t \leq x+d x$. The function $f$ is the density of probability of a continuous random variable $D D D T$. By assigning each value $R L \in\left(0, d d d t_{\max }\right)$ to its corresponding value $F(R L)$ obtained by integrating the density of probability $f(x)$ in the range from 0 to $R L$, i.e. by the rule $F(R L)=\int f(x) d x$, this paper defines the distribution function $F:\left(0, d d d t_{\max }\right) \rightarrow(0,1)$ of the continuous random variable $D D D T$ with the extreme values of $F(0)=0$ and $F\left(d d d t_{\max }\right)=1$. In the space among these extreme values, $F(R L)$ value gives the probability that $d d d t<R L$.

The discrete version of the continuous distribution function is the expression $F(R L)=\Sigma p(i)$, where it is summed over all $i<R L$. Taking into account the fact that, if summing over the entire field $\Omega, \Sigma p(i)=1$ applies, then by substituting $F(R L)$ to the equation (9), it can be obtained:

$$
1-F(R L) \geq \phi>1-F(R L)-p(R L)
$$

The condition (11) can be achieved only when

$$
\phi=1-F(R L) \text { or } F(R L)=1-\phi \text { (which says the same thing) }
$$

It is obvious that condition (12) holds for both the discrete and continuous case. What it means in the continuous case specifically is apparent from Figure 4. 


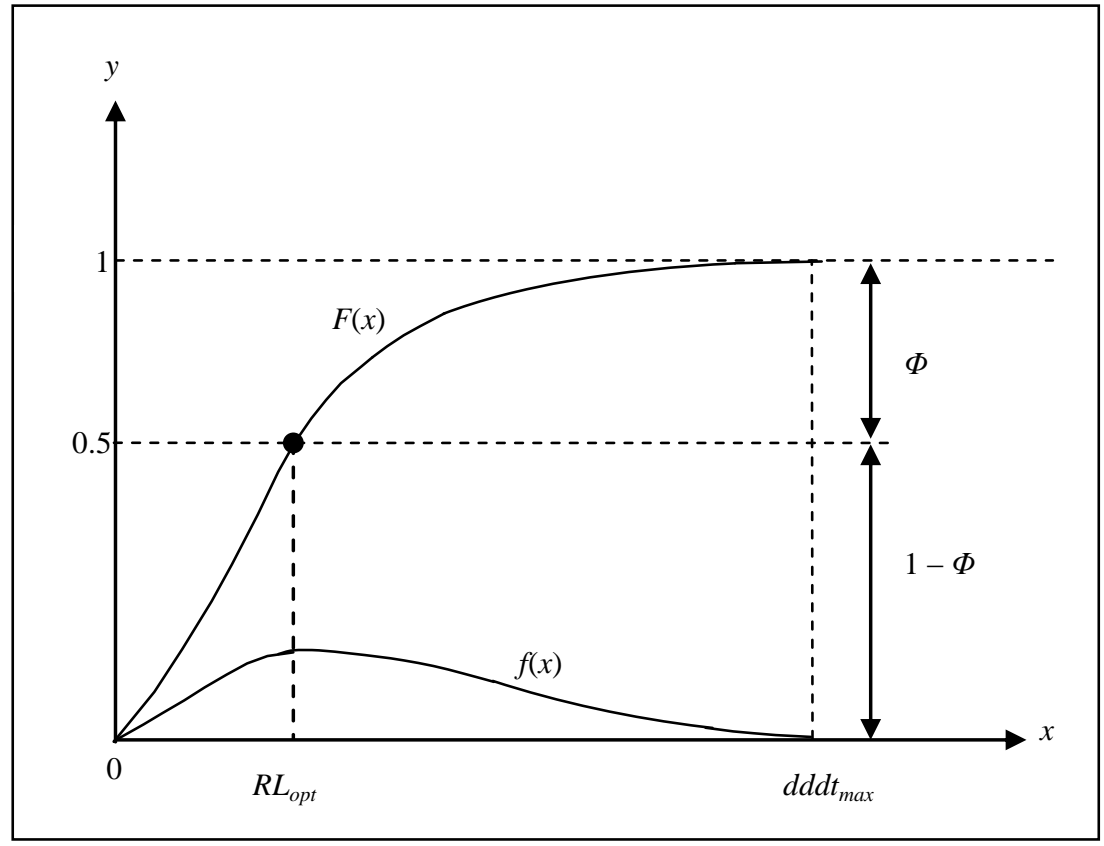

Figure 4. Identification $R L_{\text {opt }}$ from the graph of the distribution function of a continuous random variable $D D D T$.

It can be seen that the sought $R L_{\text {opt }}$ is placed under the intersection of the distribution function $F(x)$ of a random variable $D D D T$ with the level of $y=1-\Phi$.

This model can be used as a default model when contracting the logistic optimization model in the context of statistical reliability analysis (Volf \& Timková, 2014).

\section{Conclusions}

The paper focuses in detail on solving one of the fundamental problems of logistics - the questions "in what quantity" and "when" to resupply stock. While the literature gives a relatively accurate and satisfactory response in terms of certainty and uncertainty to the first question, the question "when" is often in terms of uncertainty answered more or less vaguely. The first part of the paper clarifies the standard definition of a model of the dynamics of the stock state with even storage removal in terms of certainty (Figure 1) and draws conclusions from this, which further extends and generalizes on the conditions under uncertainty.

The reality reflecting general model of the dynamics of stock in terms of uncertainty (Figure 2), which can answer the question "when", is gradually built. For this purpose, this paper first studies the discrete case in detail, the result of which is described by the algorithm of the search of the optimum by means of the flow chart in Figure 3, alternatively by the condition (9), which when converted to the condition (11) by the limit transition is also valid for the continuous case.

To answer satisfactorily the question "when" requires calculating the probability distribution of the demand during the delivery time $(D D D T)$ and the ascertainable value of, in this paper, newly established indicator of the storage efficiency. The indicator measures the loss of the dissatisfaction of the annual consumption of the inventory item and the annual storing costs of the optimum additional supply. The higher it is, the higher the safety stock level is worth holding. Its knowledge especially grows in importance in the case of the limited storage capacity indicating, which inventory items are worth storing and which are not. 
The frequently in practice occurring discreet case is presented within the solution of the model case study performing the economical procedure of the calculation of the DDDT built on the data of the analytical evidence of financial accounting. As shown, the correct result can be estimated from its several values, while the requirement on accuracy of these values is not high.

\section{References}

Brealey, R. A., Myers, S. C., \& Marcus, A. J. (2011). Fundamentals of corporate finance (7th ed.). New York: Mcgraw-Hill Education.

Chen, Y., Ray, S., \& Song, Y. (2006). Optimal pricing and inventory control policy in periodic-review systems with fixed ordering cost and lost sales. Naval Research Logistics, 53, 117-136.

Federgruen, A., \& Heching, A. (1999). Combined pricing and inventory control under uncertainty. Operation Research, 47, 454-475.

Jiroušek, R., \& Kushmerick, N. (1997). Constructing probabilistic models. International Journal of Medical Informatics, 45, 9-18.

Maddah, B., \& Jaber, M. Y. (2008). Economic order quantity for items with imperfect quality: Revisited. International Journal of Production Economics, 112, 808-815.

Shapiro, J. F., \& Wagner, S. N. (2009). Strategic inventory optimization. Journal of Business Logistics, 30, 161-173.

Volf, P., \& Timková, J. (2014). On selection of optimal stochastic model for accelerated life testing. Reliability Engineering and System Safety, 131, 291-297.

Wei, Y., \& Chen, Y. (2009). Joint determination of inventory replenishment and sales effort with uncertain market responses. International Journal of Production Economics, 134, 368-374.

Williams, B. D., \& Tokar, T. (2008). A review of inventory management research in major logistics journals: Themes and future directions. International Journal of Logistics Management, 19, 112-132.

Wokurka, R. J., \& O’Leary-Kelly, S. W. (2000). A review of empirical research on manufacturing flexibility. Journal of Operational Management, 18(4), 485-501. 